\title{
ON PARABOLICITY OF A RIEMANN SURFACE
}

\author{
MAKOTO OHTSUKA
}

In connection with [2] Professor Grunsky asked the author personally the following question at the Colloquium held at Joensuu in August, 1978.

Let $a_{1}, a_{2}, \ldots$ be a sequence of positive numbers decreasing to zero, and denote the points $-1+i a_{k}$ and $1+i a_{k}$ in the $w$-plane by $\alpha_{k}$ and $\beta_{k}$ respectively. Let $s_{k}$ be the segment $\alpha_{k} \beta_{k}$. Let $\alpha=-1$ and $\beta=1$, and $s$ the segment $\alpha \beta$. Consider an extended plane $P$ slit along $s, s_{1}, s_{2}, \ldots$, and an extended plane $P_{k}$ slit only along $s_{k}$ for $k=1,2, \ldots$. Connect $P_{k}$ crosswise with $P$ through $s_{k}$. Let $Q_{1}, Q_{2}, \ldots$ be extended planes slit along $s$, and identify the upper shore of $Q_{1}$ with the lower shore of $P$, the upper shore of $Q_{2}$ with the lower shore of $Q_{1}$, and so on. Denote by $R$ the resulting simply connected Riemann surface $P \cup P_{1} \cup P_{2} \cup \ldots \cup Q_{1} \cup Q_{2} \cup \ldots$. The question is as to whether $R$ is of parabolic type.

In this paper we shall prove

Theorem. The surface $R$ is of parabolic type.

Proof. In view of Corollaire 2 of [4, p. 201], it will be sufficient to show that the family $\Gamma$ of curves starting from a closed disk $\Delta$ in $P$ and tending to the ideal boundary of $R$ has infinite extremal length. We may assume that $\Delta$ lies above $s_{1}$. Denote by $P^{+}$(respectively $P^{-}$) the upper (respectively lower) half of $P$; let $P^{-}$include $(-\infty,-1) \cup(1, \infty)$.

Divide $\Gamma$ into four families. The first family $\Gamma_{1}$ consists of curves $c$ of $\Gamma$ such that some terminal part of $c$ is contained in $P^{-} \cup Q_{1} \cup Q_{2} \cup \ldots$. The second (respectively third) family $\Gamma_{2}$ (respectively $\Gamma_{3}$ ) consists of curves of $\Gamma$ each of which contains a sequence of points of $P^{+}$converging to $\alpha$ (respectively $\beta$ ). The fourth family $\Gamma_{4}$ consists of curves of $\Gamma-\Gamma_{2}-\Gamma_{3}$ each of which contains a sequence of points of $P^{+}$ converging to a point of $s-\{\alpha\}-\{\beta\}$. Then $\Gamma=\Gamma_{1} \cup \Gamma_{2} \cup \Gamma_{3} \cup \Gamma_{4}$.

Since $Q_{1} \cup Q_{2} \cup \ldots$ forms a "half" of the Riemann surface defined by the logarithmic function, the extremal length $\lambda\left(\Gamma_{1}\right)=\infty$. To prove $\lambda\left(\Gamma_{2}\right)=\infty$, map the part $R^{\prime}$ of $R$ lying above the left half plane $\operatorname{Re} w<0$ conformally onto the left half plane $\operatorname{Re} z<0$, and denote by $g$ the mapping function. Let $w=f(z)$ be the composition of $g^{-1}$ and the projection of $R^{\prime}$ into the $w$-plane. Take any $c \in \Gamma_{2}$ and let $\left\{w_{n}\right\}$ be a sequence of points of $c \cap P^{+}$which converges to $\alpha$ and whose image by $g$ converges to $z_{0}$ on the imaginary axis. For any $c^{\prime} \in \Gamma_{2}$ we can find a sequence $\left\{\gamma_{n}\right\}$ of arcs in $P^{+}$such that $\gamma_{n}$ connects $w_{n}$ and $c^{\prime}$ for each $n$ and its length tends to 
0 as $n \rightarrow \infty$. Suppose $f^{-1}\left(\gamma_{n}\right)$ does not tend to $z_{0}$ as $n \rightarrow \infty$. Then there exist a subsequence $\left\{f^{-1}\left(\gamma_{n_{k}}\right)\right\}$ and a sequence $\left\{c_{k}\right\}$ of arcs in $\operatorname{Re} z<0$ such that $c_{k}$ is a subarc of $f^{-1}\left(\gamma_{n_{k}}\right)$ for each $k$, one end of $c_{k}$ converges to $z_{0}$ and the other end of $c_{k}$ converges to a point $z_{0}^{\prime} \neq z_{0}$ on the imaginary $z$-axis. By applying Koebe's theorem (cf. Hilfssatz on p. 19 of [1]) we conclude that $f$ is constantly equal to $\alpha$. This is impossible. Thus the image by $g$ of $c^{\prime} \cap R^{\prime}$ contains a sequence of points tending to $z_{0}$. By symmetry $R$ is mapped conformally outside a point or a segment on the imaginary axis. The image by $g$ of $c^{\prime}$ and hence the image of every curve of $\Gamma_{2}$ contains a sequence of points tending to $z_{0}$. Therefore it converges to $z_{0}$ or is not rectifiable. It follows that $\lambda\left(\Gamma_{2}\right)=\infty$; see pp. 134-135 of [3] for a proof, for instance. Similarly $\lambda\left(\Gamma_{3}\right)=\infty$.

Finally let $\Lambda_{n}(n \geqq 2)$ be the subfamily of $\Gamma_{4}$ such that the cluster set of the part in $P$ of each curve of $\Lambda_{n}$ is contained in $(-1+1 / n, 1-1 / n) \subset s$. Evidently $\Gamma_{4}=U_{n} \Lambda_{n}$. To prove $\lambda\left(\Lambda_{n}\right)=\infty$, denote by $\alpha_{k}^{(n)}$ and $\beta_{k}^{(n)}$ the points $-1+1 / n+i a_{k}$ and $1-1 / n+i a_{k}$ respectively, and map $P_{k}$ conformally onto a rectangle $D_{k}$ of height one so that the end segments $\alpha_{k} \alpha_{k}^{(n)}$ and $\beta_{k}^{(n)} \beta_{k}$ correspond to the sides of length one; observe that $D_{1}, D_{2}, \ldots$ have the same shape. Given a curve of $\Lambda_{n}$, its image in $D_{k}$ connects opposite sides if $k$ is large. Define a density $\varrho_{k}$ in $P_{k}$ by means of the constant density $1 / k$ in $D_{k}$, and let $\varrho$ be the density on $R$ equal to $\varrho_{k}$ in $P_{k}$ for $k=$ $1,2, \ldots$ and to 0 elsewhere. Then $\int_{c} \varrho d s=\infty$ for every $c \in \Lambda_{n}$ and $\iint \varrho^{2} d x d y<\infty$. Hence $\lambda\left(\Lambda_{n}\right)=\infty$ for every $n$ so that $\lambda\left(\Gamma_{4}\right)=\infty$. Thus $\lambda(\Gamma)=\infty$.

Remark. The proof was presented at the Colloquium held at Kyoto on the occasion of Professor Lehto's visit to Japan; see [5].

\section{References}

[1] Bieberbach, L.: Lehrbuch der Funktionentheorie. Bd. II. - 2. Aufl. B. G. Teubner, Leipzig, 1931.

[2] Grunsky, H.: Analytische Fortsetzung über offene Randkomponenten einer berandeten Riemannschen Fläche. - Topics in analysis. Lecture Notes in Mathematics 419. SpringerVerlag, Berlin-Heidelberg-New York, 1974, 143-155.

[3] Lehto, O., and K. I. VIRTANEN: Quasiconformal mappings in the plane. - Die Grundlehren der mathematischen Wissenschaften 126. Springer-Verlag, Berlin-Heidelberg-New York, 1973.

[4] Ohtsuka, M.: Sur un théorème étoilé de Gross. - Nagoya Math. J. 9, 1955, 191-207.

[5] Ohtsuka, M.: On parabolicity of a Riemann surface. - Sûrikaisekikenkyûsho Kôkyûroku 364, 1979, 1-4.

Gakushuin University

Faculty of Science

Department of Mathematics

171 Tokyo

Japan

Received 11 June 1984 